\title{
Influence of exchange bias coupling on the single-crystalline FeMn ultrathin film
}

\author{
J. Wang \\ Max-Planck-Institut für Mikrostrukturphysik, Weinberg 2, D-06120 Halle, Germany, \\ and Department of Physics and HKU-CAS Joint Laboratory on New Materials, \\ The University of Hong Kong, Hong Kong, China \\ W. Kuch, ${ }^{\text {a) }}$ L. I. Chelaru, ${ }^{\text {b) }}$ F. Offi, ${ }^{\text {c) }}$ and M. Kotsugi ${ }^{\text {d) }}$ \\ Max-Planck-Institut für Mikrostrukturphysik, Weinberg 2, D-06120 Halle, Germany
}

(Received 8 September 2004; accepted 24 January 2005; published online 15 March 2005)

\begin{abstract}
Polarization dependent x-ray photoemission electron microscopy was used to investigate the influence of the exchange bias coupling on the disordered ultrathin single-crystalline fcc $\mathrm{Fe}_{50} \mathrm{Mn}_{50}$. We find that the critical thickness of the FeMn film, where the antiferromagnetic (AF) order is formed, varies with changing the magnetization direction of the ferromagnetic (FM) layer from out-of-plane to in-plane. Surface magneto-optical Kerr effect measurements (SMOKE) further manifest the shift of the critical thickness with alternating the exchange bias coupling. It indicates that the spin structure of the FeMn layer near the FM layer is modified by the presence of exchange bias coupling and the properties of the coupling. Our results provide direct experimental evidence that the AF spin structure at the interface between the FM and AF layers is strongly influenced by the exchange bias coupling. (C) 2005 American Institute of Physics. [DOI: 10.1063/1.1883318]
\end{abstract}

Investigations of exchange coupling between antiferromagnetic (AF) and ferromagnetic (FM) materials have received much attention recently due to its important applications in the data storage industry, ${ }^{1,2}$ such as magnetoresistive sensors and read heads as well as magnetic random access memory. Theoretical and experimental progresses have been made for understanding the mechanism of the exchange bias effect. $^{3-11}$ In spite of efforts during the past 40 years, there is still difficulty in theoretically relating the observed bias to the actual interface coupling. The lack of knowledge about the real AF spin structure in ultrathin AF/FM bilayers is one of the main reasons to the difficulty. The spin structure of the relevant AF bulk materials is often assumed in understanding the underlying principles governing the manifestation of exchange bias coupling. However, the influence of the exchange coupling on the AF spin structure in ultrathin AF/FM bilayers should also be considered, which was rarely discussed in most reports. It has been shown that recently the AF spin structure in a $\mathrm{NiO}$ (001) single crystal near its interface with $\mathrm{Co}$ or Fe may significantly deviate from that in the bulk antiferromagnet. ${ }^{12}$ Moreover, the influence of the different type of coupling (with perpendicular or in-plane anisotropy FM layer) on the spin structure of ultrathin AF films may be expected. The faced centered cubic (fcc) $\gamma-\mathrm{Fe}_{50} \mathrm{Mn}_{50}$ single crystal films have been widely used in so-called spin-valve structures as a pinning layer. So far three theoretical models of magnetic structures in disordered bulk FeMn have been proposed: ${ }^{13-16}$ a collinear AF (1Q) structure and two noncollinear 2Q and 3Q structures, shown in Fig. 1. By using the linearized muffin-tin orbital (LMTO) method,

\footnotetext{
a) Present address: Freie Universität Berlin, Institut für Experimentalphysik, Arnimallee 14, D-14195 Berlin, Germany.

${ }^{b)}$ Present address: Universität Duisburg-Essen, Institut für Experimentelle Physik, Universitätsstrasse 5, D-45117 Essen, Germany.

${ }^{c)}$ Present address: INFM, Unità Roma Tre, Via della Vasca Navale 84, I-00146 Roma, Italy.

${ }^{\text {d) }}$ Present address: Hiroshima Synchrotron Radiation Center, 2-313 Kagamiyama, Higashi-Hiroshima 739-8526, Japan.
}

Spišák and Hafner reported that the presence of disorder stabilizes 1Q nearly ideal collinear layered AF spin structure. ${ }^{13}$ Other theoretical calculations show that the noncollinear $3 \mathrm{Q}$ and $2 \mathrm{Q}$ structures are both energetically favorable with the former being slightly lower in energy, ${ }^{14}$ so that any small disturbance could favor one or the other spin structure.

More recently, the x-ray magnetic circular dichroism photoelectron emission microscopic (XMCD-PEEM) studies of $\mathrm{Co} / \mathrm{FeMn}$ bilayers have been reported. Appearance of very small domains and rotation of the Co magnetization above a critical thickness of 10 atomic monolayers (ML) due to the exchange-bias coupling was observed in $\mathrm{Co} / \mathrm{FeMn}$ and $\mathrm{FeMn} / \mathrm{Co}$ bilayers respectively, ${ }^{17,18}$ which was ascribed to the pinning by locally uncompensated $\mathrm{AF}$ spins at the surface of the FeMn layer.

In this letter we present an element-resolved XMCDPEEM study of single crystalline $\mathrm{Fe}_{50} \mathrm{Mn}_{50}$ in contacting with FM layer with out-of-plane or in-plane magnetization. $\mathrm{Ni} / \mathrm{FeMn}$ and $\mathrm{Co} / \mathrm{Ni} / \mathrm{FeMn} / \mathrm{Ni}$ double wedge samples were grown at room temperature on $\mathrm{Cu}(001)$ by electron beam assisted thermal evaporation under zero magnetic field. All the evaporations and measurements were performed with the base pressure below $5 \times 10^{-10}$ mbar. $\mathrm{Fe}_{50} \mathrm{Mn}_{50}$ films were obtained by coevaporation of $\mathrm{Fe}$ and Mn from two different

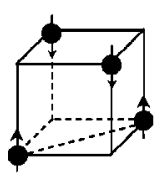

(a) $1 \mathrm{Q}$

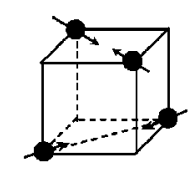

(b) $2 Q$

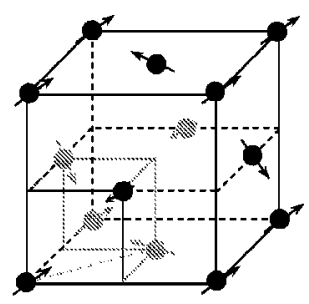

(c) $3 Q$
FIG. 1. Schematic of the magnetic structures of disorder $\mathrm{Fe}_{50} \mathrm{Mn}_{50}$ : (a) noncollinear 3Q; (b) noncollinear 2Q, and (c) 1Q collinear spin configurations. Arrows indicate the spin directions. 


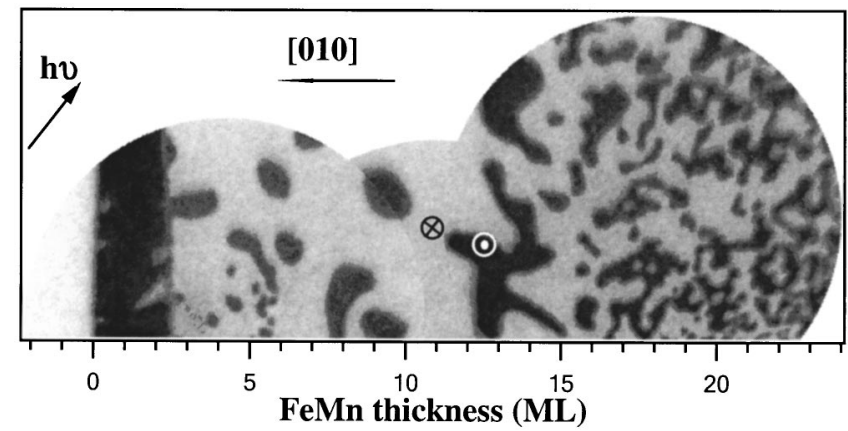

FIG. 2. As-grown Ni domain image of $15 \mathrm{ML} \mathrm{Ni/FeMn} \mathrm{wedge/Cu(001).}$ The FeMn thickness increases from left to right indicated at the bottom axis.

sources. Film thicknesses were calibrated by oscillations of the diffracted medium energy electron intensity during evaporation. The systematic error of the cited thickness is about $10 \%$ for FeMn and Ni. However, the accuracy of the relative thickness within the same sample is about $1 \%$. The FeMn and bottom Ni layer were grown as crossed wedges with $155 \mu \mathrm{m}$ width. Circularly polarized $\mathrm{x}$ rays from the helical undulator beamline UE56-2 PGM2 of BESSY II in Berlin were used, incident to the sample under an angle of $60^{\circ}$ from the surface normal. The lateral resolution and field of view were set to $400 \mathrm{~nm}$ and $90 \mu \mathrm{m}$, respectively. The detailed description of the setup and operation of this PEEM can be found in an earlier paper. ${ }^{19}$

Figure 2 shows the as-grown $\mathrm{Ni}$ domain image of 15 atomic monolayers (ML) Ni on top of a wedge shaped FeMn film. The FeMn thickness increases from left to right (0-20 ML), as indicated at the bottom axis. Measurements under different light incidence azimuth show that the magnetization of the domains in this sample is alternately pointing out and into the film plane as indicated by arrows in Fig. 2. The observed perpendicular magnetization direction of the $\mathrm{Ni} / \mathrm{FeMn}$ bilayers is similar to previous reports on the $\mathrm{Ni}$ films. ${ }^{20}$ Small irregular domains with an average size depending on the FeMn thickness can be observed. Between 0 and 3 ML FeMn a rectangular-like domain with black contrast appears in Ni. With the thickness of FeMn layer increases further, small domains are formed gradually, and many ones appear in the region where the thickness of FeMn is thicker than $15 \mathrm{ML}$, which is attributed to the coupling from the antiferromagnetic FeMn film. The local pinning from the domains in the FeMn layer results in the collapse of big domain in $\mathrm{Ni}$, the small domains are energetically favored. However, unlike the case in $\mathrm{Co} / \mathrm{FeMn}$ bilayer, ${ }^{17}$ in the image we have not found a critical thickness of FeMn layer (i.e., the AF order formed thickness at room temperature) where the very small domains are formed suddenly. It implies that the properties of the FeMn layer are probably different in $\mathrm{Co} / \mathrm{FeMn}$ and Ni/FeMn bilayers.

Figure 3 shows the domain images obtained at the $\mathrm{Ni} L_{3}$ edge of a $\mathrm{Co} / \mathrm{Ni} / \mathrm{FeMn} / \mathrm{Ni}$ trilayer in which both of the $\mathrm{Ni}$ layers are $15 \mathrm{ML}$ and a wedge shaped FeMn layer was sandwiched between them. The bottom Ni layer was annealed to $450 \mathrm{~K}^{21}$ and saturated by an out-of-plane 500 Oe external field. The top Co layer was deposited as also a wedge with orientation perpendicular to the FeMn wedge. In the bottom part of Fig. 3, where the Co thickness is below 0.5 ML, a periodic alternating dark and down out-of-plane stripes with 2 ML period were observed above $7 \mathrm{ML}$ FeMn, which is due

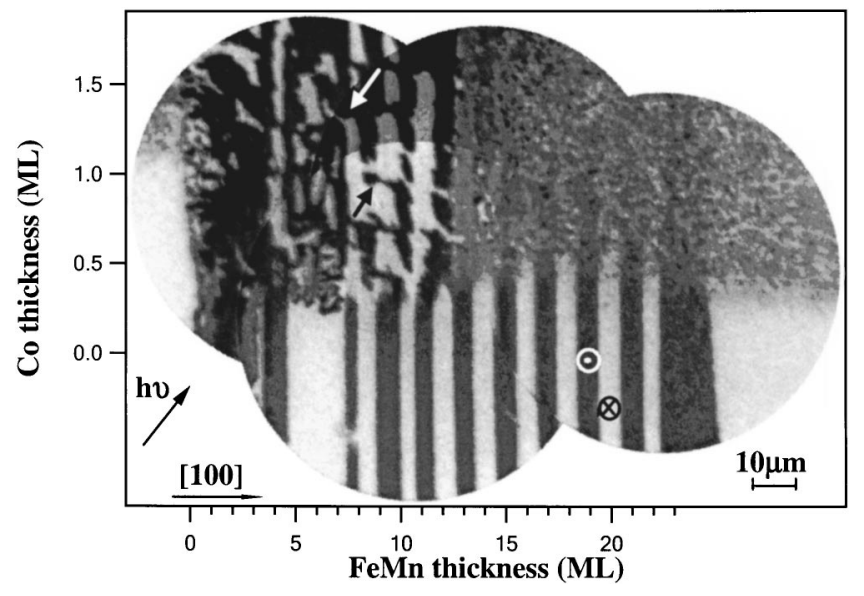

FIG. 3. Magnetic domain image at the $\mathrm{Ni} L_{3}$ edge of $\mathrm{Co} / \mathrm{Ni} / \mathrm{FeMn} / \mathrm{Ni} / \mathrm{Cu}(001)$. The FeMn and Co layers are crossed wedge, and their thickness is indicated at the bottom and left axis, respectively. Both Ni layers are $15 \mathrm{ML}$

to the magnetic exchange coupling between the two Ni layers. The presence of the oscillation might be related with the ordered AF state of FeMn. Actually, we will show below that $8 \mathrm{ML}$ is probably the ordering thickness for FeMn in $\mathrm{Ni}-$ FeMn systems. When the Co layer thickness is above 0.5 ML, the direction of the Ni magnetization changes from outof-plane to in-plane as verified by images taken under opposite light incidence azimuth. Some small domains appear in the region below $12 \mathrm{ML}$ of the FeMn layer. One can see some correlation between the $\mathrm{Ni}$ out-of-plane stripe-like magnetic domains structure before $\mathrm{Co}$ deposition and the in-plane domains. Analysis on the comparison of these inplane domains gives the magnetization direction of [110] and $[-1-10] .{ }^{17,22}$ For the FeMn thickness above 12 ML very small domains, most likely magnetized in-plane, are formed in the top FM layer. One could also observe a change of the domain contrast with the FeMn thickness across $12 \mathrm{ML}$, indicating the magnetization turns from [110] to $[100] .{ }^{17,22} \mathrm{It}$ means that the AF ordering thickness shifts to $12 \mathrm{ML} .^{17,18}$ The change of the FeMn spin structure due to the change of the coupling between the top Ni layer and FeMn layer might be the reason of this shift.

Surface magneto-optical Kerr effect measurements (SMOKE) experiments give further evidence of this. Figure 4 shows the dependence of coercivity $\left(H_{C}\right)$ and the normalized Kerr signal in remanence $\left(M_{r}\right)$ on the thickness of FeMn layer $\left(t_{\mathrm{FeMn}}\right)$. It is found that $H_{c}\left(M_{r}\right)$ of the sample with a FeMn layer thicker than $3 \mathrm{ML}$ is a bit lower (higher) than the one of the sample without FeMn layer, which is the contribution from induced moments in FeMn layer thinner than 3 ML. ${ }^{22}$ With the FeMn thickness above $8 \mathrm{ML}$, an enhancement of $H_{c}$ originating from the pinning by AF FeMn is observed, which indicates that the AF order of the FeMn layer in FeMn/Ni bilayer is established at this thickness. Comparing with the results in FeMn/Co bilayer, we found a 2 ML difference of the magnetic order transition thickness in FeMn films. The data of FeMn/6-MLCo is presented in the inset of Fig. 4. It is seen that the pronounced increase of $H_{c}$ (more than 2 times) in FeMn/Ni occurs at $8 \mathrm{ML}$, while this increase starts from $10 \mathrm{ML}$ in FeMn/Co (inset of Fig. 3). To rule out the effect of the deviation of FeMn thickness caused by the bigger interfacial roughness in FeMn/Ni compared to FeMn/Co, we annealed the sample to $450 \mathrm{~K}^{21}$ before depos- 


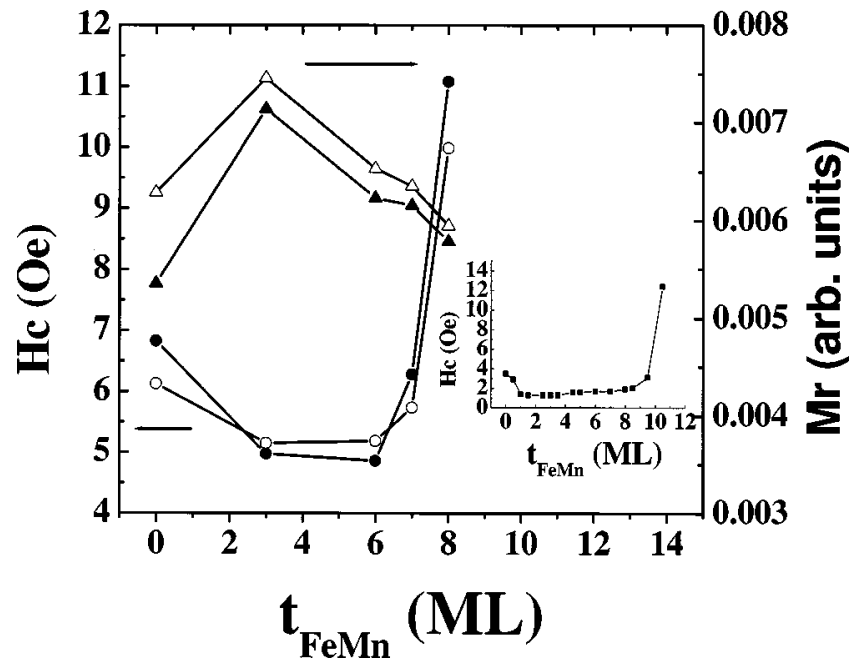

FIG. 4. Coercivity field $H_{c}$ (left axis) and remanence $M_{r}$ (right axis) as a function of $\mathrm{Fe}_{50} \mathrm{Mn}_{50}$ film thickness $\left(t_{\mathrm{FeMn}}\right)$ at room temperature for $\mathrm{Fe}_{50} \mathrm{Mn}_{50} / 15-\mathrm{ML}-\mathrm{Ni} / \mathrm{Cu}(001)$. The external field was applied along the normal direction of the sample. The inset displays the FeMn layer thickness dependence of coercivity field $H_{c}$ for $\mathrm{Fe}_{50} \mathrm{Mn}_{50} / 6$ ML Co.

iting the FeMn layer. The data are also plotted in Fig. 4 (filled points). As one can see, the same critical thickness is obtained.

These results probably indicate that the exchange coupling between AF and FM layers influence not only the magnetic properties of the FM film but also the AF film. On the other hand, the different interfacial interaction for $\mathrm{Co} / \mathrm{FeMn}$ and $\mathrm{Ni} / \mathrm{FeMn}$ may also contribute more or less to the different FeMn critical thickness. The present SMOKE results could not give related information. However, one could find some hints from the domain image of $\mathrm{Co} / \mathrm{Ni} / \mathrm{FeMn} / \mathrm{Ni}$ as shown in Fig. 3. As we discussed above, the exchange coupling-induced regular vertical stripes appear above a FeMn thickness of about $7 \mathrm{ML}$. The appearance of the stripes should be related to the formation of the AF order. One can find it is coordinating to the critical thickness obtained by SMOKE. However, with 0.5 ML Co deposited on the top of the sample, the critical thickness of the FeMn moves to $12 \mathrm{ML}$ as discussed above. It indicates that the change of the magnetization direction in the ferromagnetic layer do affect the properties of the FeMn layer. To completely rule out the influence of different interfacial situation and solely pick out the relationship between the AF order and the spin direction of the FM layer, more detailed work are still required.

Some theoretical calculations and experiments have pointed out that the $3 \mathrm{Q}$ noncollinear structure is the most energetically favored for the ground state. ${ }^{14-16}$ When a FM layer is in contact with the FeMn layer, the exchange bias coupling will be established, resulting in the enhancement of the coercivity and the decrease of the average as-grown domain size. On the other hand, this coupling will also affect the spin structure of the FeMn layer. This effect is controlled by the magnetization direction of the FM layer. Through the longitude coupling a FM layer with in-plane magnetization will force the spin of the FeMn layer tilt towards in-plane, resulting in the spin structure of the FeMn layer deviating from $3 \mathrm{Q}$ to $2 \mathrm{Q}$. A new energy balance may be reached at some state between $3 \mathrm{Q}$ and $2 \mathrm{Q}$, which is dependent on the relative strength of the exchange coupling. However, when the FeMn layer is deposited on top of an out-of-plane Ni film or sandwiched between two out-of-plane Ni film, a perpendicular coupling from the interface $\mathrm{Ni}$ spins causes the spins of FeMn layer tilt to the vertical direction, i.e., towards 1Q. The difference of the spin structure between the two cases results in the different AF ordering thickness in FeMn layer. It is worthy to point out the spin structure of the FeMn layer in all the cases we discussed is still a three-dimensional spin structure. ${ }^{23}$ The deviation from the $3 \mathrm{Q}$ spin structure due to the coupling from FM layer is not sufficient to establish 2Q or 1Q structure.

In summary, we investigate the influence of the exchange coupling between the FeMn layer and FM layer on the properties of the FeMn layer. We observe a different critical thickness in the ultrathin FeMn layers in contact with in-plane and out-of-plane FM layers. This was explained by the deviation of the interface FeMn spin structure from the bulk one due to the exchange coupling from the FM layers. Our results indicate both the ferromagnetic and antiferromagnetic spin orientations are sensitive to the coupling properties in exchange bias systems. The realistic models for the exchange bias should take into account the actual spin configuration near the interface, which may significantly deviate from the bulk one.

The authors thank F. Helbig, B. Zada, and W. Mahler for technical assistance. Financial support by BMBF (No. 05KS1EFA6) is gratefully acknowledged.

${ }^{1}$ B. Dieny, V. S. Speriosu, S. S. P. Parkin, B. A. Gurney, D. R. Wilhoit, and D. Mauri, Phys. Rev. B 43, 1297 (1991); J. C. S. Kools, IEEE Trans. Magn. 32, 3165 (1996).

${ }^{2}$ J. O. Oti and S. E. Russek, IEEE Trans. Magn. 33, 3298 (1997).

${ }^{3}$ For a recent review, see, J. Nogués and I. K. Schuller, J. Magn. Magn. Mater. 192, 203 (1999).

${ }^{4}$ J. V. Kim, R. L. Stamps, B. V. McGrath, and R. E. Camley, Phys. Rev. B 61, 8888 (2000).

${ }^{5}$ D. Mauri, H. C. Siegmann, P. S. Bagus, and E. Key, J. Appl. Phys. 62, 3047 (1987).

${ }^{6}$ A. P. Malozemoff, Phys. Rev. B 35, 3679 (1987)

${ }^{7}$ N. C. Koon, Phys. Rev. Lett. 78, 4865 (1997).

${ }^{8}$ T. C. Schultness and W. H. Butler, Phys. Rev. Lett. 81, 4516 (1998).

${ }^{9}$ C. -M. Park, K. -I. Min, and K. H. Shin, J. Appl. Phys. 79, 6228 (1996); J. Nogués, T. J. Moran, D. Lederman, I. K. Schuller, and K. V. Rao, Phys. Rev. B 59, 6984 (1999).

${ }^{10}$ V. I. Nikitenko, V. S. Gornakov, A. J. Shapiro, R. D. Shull, K. Liu, S. M. Zhou, and C. L. Chien, Phys. Rev. Lett. 84, 765 (2000).

${ }^{11}$ K. Takano, R. H. Kodama, A. E. Berkowitz, W. Cao, and G. Thomas, Phys. Rev. Lett. 79, 1130 (1997).

${ }^{12}$ H. Ohldag, A. Scholl, F. Nolting, S. Anders, F. U. Hillebrecht, and J. Stöhr, Phys. Rev. Lett. 86, 2878 (2001).

${ }^{13}$ D. Spišák and J. Hafner, Phys. Rev. B 61, 11569 (2000).

${ }^{14}$ T. C. Schulthess, W. H. Butler, G. M. Stocks, S. Maat, and G. J. Mankey, J. Appl. Phys. 85, 4842 (1999).

${ }^{15}$ S. J. Kennedy and T. J. Hicks, J. Phys. F: Met. Phys. 17, 1599 (1987).

${ }^{16}$ A. Sakuma, J. Phys. Soc. Jpn. 69, 3072 (2000).

${ }^{17}$ W. Kuch, F. Offi, L. I. Chelaru, M. Kotsugi, K. Fukumoto, and J. Kirschner, Phys. Rev. B 65, 140408R (2002).

${ }^{18}$ F. Offi, W. Kuch, and J. Kirschner, Phys. Rev. B 66, 064419 (2002).

${ }^{19}$ W. Kuch, R. Frömter, J. Gilles, D. Hartmann, Ch. Ziethen, C. M. Schneider, G. Schönhense, W. Swiech, and J. Kirschner, Surf. Rev. Lett. 5, 1241 (1998).

${ }^{20}$ T. C. Schultness, W. H. Bulter, G. M. Stocks, S. Maat, and G. J. Mankey, J. Appl. Phys. 85, 4842 (1999).

${ }^{21}$ J. Shen, J. Giergiel, and J. Kirschner, Phys. Rev. B 52, 8454 (1995); J. Shen, M.-T. Lin, J. Giergiel, C. Schmidthals, M. Zharnikov, C. M. Schneider, and J. Kirschner, J. Magn. Magn. Mater. 156, 104 (1996).

${ }^{22}$ F. Offi, W. Kuch, L. I. Chelaru, K. Fukumoto, M. Kotsugi, and J. Kirschner, Phys. Rev. B 67, 094419 (2003).

${ }^{23}$ W. Kuch, L. I. Chelaru, F. Offi, J. Wang, M. Kotsugi, and J. Kirschner, Phys. Rev. Lett. 92, 017201 (2004). 